\title{
PRODUTIVIDADE ACUMULADA COMO CRITÉRIO DE SELEÇÃO EM FÊMEAS DA RAÇA NELORE
}

\author{
ACCUMULATED PRODUCTIVITY AS SELECTION CRITERIA IN NELLORE \\ BREED FEMALES
}

\author{
Eduardo Brum Schwengber ${ }^{1}$ Luiz Antônio Framartino Bezerra ${ }^{2}$ Raysildo Barbosa Lôbo ${ }^{3}$
}

RESUMO

O presente trabalho teve por objetivo determinar os componentes de variância e estimar a herdabilidade da produtividade acumulada (PAC) de 15.070 fêmeas, criadas em diferentes rebanhos participantes do Programa de Melhoramento Genético da Raça Nelore. A PAC é um índice que considera a produção total de bezerros desmamados em $\mathrm{kg}$, o tempo total de produção de bezerros e o início de parição. As análises estatísticas foram realizadas por meio do programa SAS (Statistical Analysis System) e os componentes de variância pelo método de máxima verossimilhança restrita utilizando o software MTDFREML. A média da PAC foi de $130 \mathrm{~kg}$ de bezerros desmamados por vaca ao ano, e os efeitos do pai da vaca, rebanho e ano de nascimento da vaca foram significativos $(P<0,0001)$ na variação dessa característica. $O$ coeficiente de herdabilidade da PAC foi estimado em 0,15 , indicando a existência de variabilidade genética suficiente para sua inclusão nos programas de melhoramento, o que resultaria na obtenção de fêmeas mais produtivas nos rebanhos.

Palavras-chave: herdabilidade, produtividade, raça Nelore.

\section{SUMMARY}

This work had for objective to determine the variance components and to estimate the heritability of the accumulated productivity (ACP) of 15,070 females, reared in different participant herds of the Nellore Breeding Program. ACP is an index that considers the total production of calves weaned in $\mathrm{kg}$, the total time of production of calves and the calving beginning. The statistical analyses were accomplished through the SAS program (Statistical Analysis System) and the variance components for the restricted maximum likelihood method using the software MTDFREML. The average of ACP was of $130 \mathrm{~kg}$ of calves weaned by cow to the year, and the sire of cow effects, herd and year of the birth cow significatly $(P<0.0001)$ affected in the variation of this characteristic. The coefficient of heritability of ACP was estimated in 0.15, indicating the existence of enough genetic variability for its inclusion in the improvement programs, what would result in the obtaining of more productive females in the herds.

Key words: heritability, productivity, Nellore breed.

\section{INTRODUÇÃO}

Nos trópicos, a baixa fertilidade dos bovinos de corte é uma limitação principal da produtividade dos rebanhos, sendo que a eficiência produtiva pode ser melhorada desde que se identifiquem as fêmeas de maior rentabilidade. É necessário, portanto, determinar os fatores que contribuem para a eficiência do ciclo de vida da vaca e sua importância relativa. O que se busca é o equilíbrio entre os fatores que determinam a fertilidade, pois essa, aliada à precocidade, eleva a taxa de nascimento do rebanho, auxiliando o processo de seleção, assim como o aumento do desfrute, resultando em maior quantidade de carne produzida ao ano e, conseqüentemente, maior rentabilidade ao criador.

Diferentes métodos para avaliar a produtividade de vacas foram estudados em bovinos de corte (DINKEL \& BROWN, 1978; EUCLIDES FILHO et al., 1984; KIRKPATRICK et al., 1985; RAHNEFELD et al., 1993), utilizando características do bezerro e da matriz, comprovando que o peso

\footnotetext{
${ }^{1}$ Zootecnista, Professor da Faculdade de Zootecnia, Veterinária e Agronomia de Uruguaiana - PUCRS e Doutorando do Departamento de Genética, Bloco C, Faculdade de Medicina de Ribeirão Preto, USP, Av. Bandeirantes 3900, 14040-030, Ribeirão Preto, SP. E-mail: edbrum@pucrs.campus2.br. Autor para correspondência.

${ }^{2}$ Analista de Sistema, MSc., Departamento de Genética da Faculdade de Medicina de Ribeirão Preto - USP.

${ }^{3}$ Médico Veterinário, PhD., Bolsista do CNPq, Professor Associado da Faculdade de Medicina de Ribeirão Preto - USP. Recebido para publicação em 08.06.00. Aprovado em 04.10.00
} 
do bezerro ao desmame é o melhor preditor. Vários estudos obtiveram parâmetros genéticos para o peso do bezerro ao desmame (MERCADANTE, 1995; FERRAZ FILHO et al., 1998; GARNERO, 1999).

O Programa de Melhoramento Genético da Raça Nelore (PMGRN), visando a viabilizar um modelo prático para o produtor aumentar a produtividade do rebanho, no início da década de 90, criou uma fórmula que contabiliza simultaneamente a fertilidade e a produção de carne, denominada fertilidade real (FR=PBDx365/Intervalo de partos), que expressa kg de bezerros desmamados (PBD) por ano efetivo (LÔBO, 1996).

Estudando a lucratividade do sistema de produção, BITTENCOURT et al. (1997), utilizando o índice de fertilidade real, verificaram que os mais lucrativos foram aqueles que obtiveram melhor relação entre o desempenho reprodutivo e a habilidade maternal da vaca e que, portanto, devem ser incluídos em programas de melhoramento genético de gado de corte. O objetivo da construção de um índice é combinar todos os registros relevantes de uma matriz em um único valor que expresse o seu mérito, e a fórmula fertilidade real traz o inconveniente de incluir o intervalo de partos e não contempla as fêmeas que parem uma vez ou mesmo aquelas que não o fazem.

Segundo MERCADANTE (1995), fórmulas que visam a avaliar a produtividade total devem ser utilizadas com certa precaução, pois incorporam muitas características de diferentes herdabilidades e importância econômica. A herdabilidade dessas tende a ser controlada pela característica mais variável e menos herdável, considerada na equação NOTTER (1995).

Nessas condições o PMGRN propôs um índice que visa a avaliar a produtividade de fêmeas, denominado produtividade acumulada (PAC), a qual considera a produção total de bezerros desmamados (em $\mathrm{kg}$ ), o tempo total de produção de bezerros e o início de parição (LÔBO,1996). Ele refere-se à contribuição da matriz durante sua permanência no rebanho e, portanto, ligada diretamente à eficiência reprodutiva, sendo dependente da idade ao primeiro parto e intervalo de partos. Em estudo recente, ROSA (1999) constatou efeito significativo da fazenda e ano de nascimento da vaca sobre essa característica.

O objetivo do presente estudo foi estimar os componentes de variância e determinar a herdabilidade da produtividade acumulada, averiguando a viabilidade da seleção de matrizes com base nessa característica.

\section{MATERIAL E MÉTODOS}

As informações utilizadas neste estudo são provenientes do Programa de Melhoramento Genético da Raça Nelore (PMGRN), coordenado pelo Departamento de Genética da Faculdade de Medicina de Ribeirão Preto - USP. Os registros utilizados neste estudo pertencem a 15.070 fêmeas, nascidas no período de 1976 a 1995, e criadas em 65 rebanhos, localizados em diferentes regiões do País, onde o clima predominante varia de Subtropical Quente Úmido a Tropical Quente Úmido, apresentando duas estações definidas, sendo que o período seco do ano compreende os meses de abril a setembro. Os animais são criados a pasto, e o manejo reprodutivo compreende uma estação de acasalamento que varia de 60 a 90 dias de duração, e a maioria dos rebanhos utiliza a inseminação artificial, com repasse de touros, após o primeiro ou segundo serviço.

A Produtividade Acumulada (PAC) foi obtida pela aplicação da expressão citada por Lôbo (1996), representada como:

$$
P A C=\frac{\bar{P}_{d} \times n_{p} \times 365}{I V P_{n}-550}
$$

sendo: PAC $=$ produtividade acumulada $; \mathrm{P}_{\mathrm{d}}=$ peso médio dos bezerros a desmama; $\mathrm{n}_{\mathrm{p}}=$ número total de bezerros produzidos; $\mathrm{IVP}_{\mathrm{n}}=$ idade da vaca ao último parto.

O programa SAS (Statistical Analysis System, 1995) foi utilizado para a análise estatística da característica em estudo, segundo o modelo descrito a seguir: $\mathbf{Y}_{\mathbf{i j k}}=\boldsymbol{\mu}+\mathbf{a}_{\mathbf{i}}+\mathbf{F}_{\mathbf{j}}+\mathbf{e}_{\mathbf{i j k}}$ no qual,

$\mathrm{Y}_{\mathrm{ijk}}=$ resposta da produtividade acumula$\mathrm{da} ; \mu=$ média geral; $\mathrm{a}_{\mathrm{i}}=$ efeito do pai da vaca (aleatório); $\mathrm{F}_{\mathrm{j}}=$ conjunto de efeitos fixos (fazenda, ano e época de nascimento da vaca); e $\mathrm{e}_{\mathrm{ijk}}=$ erro aleatório associado a cada observação.

Os efeitos fixos considerados na formação dos grupos de contemporâneos consistiram de fazenda, ano e época de nascimento da vaca. A época de nascimento da vaca compreendeu os meses de outubro a março, como sendo a estação das águas e os meses de abril a setembro, a estação seca.

Os componentes de variância genética e residuais foram estimados pelo método de máxima verossimilhança restrita, pela aplicação do software MTDFREML (BOLDMAN $\boldsymbol{e}$ t $\boldsymbol{a l}$., 1995), de acordo com o modelo misto descrito a seguir: 


\section{$\mathbf{Y}=\mathbf{X} \beta+\mathbf{Z}_{\mathbf{1}} \mathbf{a}+\mathbf{e}$}

sendo: $\mathrm{Y}=$ vetor de observações da característica; $\mathrm{X}$ $=$ matriz de incidência dos efeitos fixos; $\beta=$ vetor dos efeitos fixos; $Z_{1}=$ matriz de incidência do efeito genético direto de cada animal; $a=$ vetor de efeitos genéticos diretos aleatórios; $\mathrm{e}=$ vetor de efeitos residuais aleatórios.

As pressuposições acerca da distribuição de $y, a$, e $\varepsilon$ são descritas como:

$$
\left[\begin{array}{l}
y \\
a \\
\varepsilon
\end{array}\right] \approx N M V\left\{\left[\begin{array}{l}
X \beta \\
0 \\
0
\end{array}\right]\left[\begin{array}{ccc}
V & Z G & R \\
G Z^{\prime} & G & 0 \\
R & 0 & R
\end{array}\right]\right\}
$$

\section{RESULTADOS E DISCUSSÃO}

A média da produtividade acumulada e o respectivo desvio padrão das fêmeas do PMGRN foram $130 \pm 35 \mathrm{~kg}$ de bezerros desmamados por vaca ao ano, inferior ao reportado por ROSA (1999) que era $144 \mathrm{~kg}$. Esse autor, porém, analisou a PAC em número inferior de fêmeas em relação ao presente estudo. A média da PAC, por se tratar de valor anual e efetivo por fêmea, está em concordância com a média do peso ao desmame do PMGRN que é de $191 \mathrm{~kg}$ e não leva em consideração o tempo que cada mãe levou para produzi-lo.

O pai da vaca teve influência significativa na variação da PAC $(\mathrm{P}<0,0001)$, conforme se pode observar na tabela 1 , onde é apresentado o resumo da análise de variância. Isso representa o reflexo na variação entre touros para o peso do bezerro ao desmame, uma vez que as mães herdaram genes para habilidade materna e que irão interferir no crescimento de sua progênie. Isso nos indica que existem diferenças nos valores genéticos na expressão da característica.

Tabela 1 - Resumo da análise de variância da produtividade acumulada (PAC) pelo método dos quadrados mínimos.

Fonte de variação Graus de liberdade Quadrado médio

\begin{tabular}{lrr}
\hline & 1505 & $1389,52^{* *}$ \\
Pai & 63 & $42127,69^{* *}$ \\
Ano & 19 & $3279,60^{* *}$ \\
Época de nascimento & 1 & $136,48^{\mathrm{ns}}$ \\
\hline
\end{tabular}

** = significativo $(\mathrm{P}<0,0001)$

${ }^{\mathrm{ns}}=$ não significativo
A fazenda e o ano de nascimento da vaca apresentaram efeitos significativos $(\mathrm{P}<0,0001)$ na variação da PAC (Tabela 1), concordando aos obtidos por ROSA (1999). A média da PAC variou, segundo os rebanhos, de $64 \mathrm{~kg}$ a $173 \mathrm{~kg}$, sendo essas diferenças devido às alterações provocadas pelas condições climáticas de cada região e ou às práticas de manejo provocadas pelo próprio criador. Aliandose a esse fato, pode-se também citar a diversidade genética dos animais como resposta aos métodos de seleção aplicados nos diferentes rebanhos.

Observa-se tendência linear crescente nas médias da PAC em função do ano de nascimento da vaca (Figura 1), provavelmente devido ao maior volume de informações disponíveis, ao melhor controle dos dados referentes à reprodução das fêmeas pelo criador, ao nível genético dos rebanhos ao longo dos anos como resposta à seleção para maior peso ao desmame e menor idade ao primeiro parto que traz, como consequiência, o maior número de crias por fêmea acasalada.

A época de nascimento da vaca não interferiu na variação da PAC, provavelmente porque esse índice é elaborado levando em consideração os pesos dos bezerros ao desmame e que ocorre em estações diferentes, segundo a ordem do parto e, portanto, pouca importância teria no desenvolvimento das crias.

Os componentes de variância e a estimativa de herdabilidade para a característica produtividade acumulada estão representados na tabela 2 . O coeficiente de herdabilidade da PAC, neste estudo, foi de magnitude semelhante ao estimado por ROSA (1999) em 0,19. A herdabilidade da PAC em 0,15 decorre do fato de ser essa variável composta por outras características e que são de caráter reprodutivo e, portanto, de herdabilidade inferior, quando comparada às características de produção, reforçando a citação de NOTTER (1995). Por outro lado, a magnitude da herdabilidade estimada da PAC é considerada superior em relação àquelas encontradas

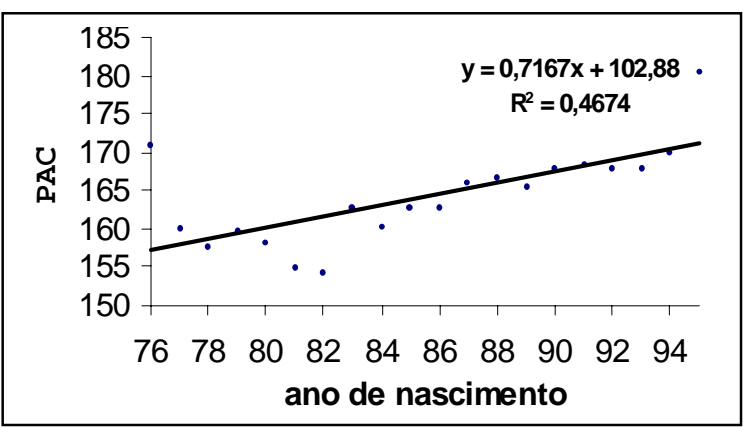

Figura 1 - Coeficiente de regressão da produtividade acumulada (PAC) em função do ano de nascimento da vaca. 
Tabela 2 - Estimativa dos componentes de variância e herdabilidade da característica produtividade acumulada (PAC).

\begin{tabular}{lcccc}
\hline Característica & $\sigma_{\mathrm{a}}^{2}$ & $\sigma_{\mathrm{e}}^{2}$ & $\sigma_{\mathrm{f}}^{2}$ & $\mathrm{~h}^{2}$ \\
PAC & 115,10 & 639,45 & 754,55 & 0,15 \\
\hline
\end{tabular}

PAC $=$ produtividade acumulada $; \sigma^{2}=$ variância genética aditiva; $\sigma_{\mathrm{e}}^{2}=$ variância residual; $\sigma_{\mathrm{f}}^{2}=$ variância fenotípica; $h^{2}=$ coeficiente de herdabilidade.

para as características reprodutivas, que, segundo MERCADANTE (1995), para as variáveis fertilidade real e intervalo de partos foram de 0,10. Esse resultado indica a existência de variabilidade genética aditiva da PAC suficiente para ser incluída em um programa de seleção.

$\mathrm{O}$ peso do bezerro ao desmame reflete a capacidade da matriz de cuidar da cria e a sua produção de leite, e os valores de herdabilidade citados por MERCADANTE (1995) em 0,29, FERRAZ FILHO et al. (1998) em 0,28 e GARNERO (1999) em 0,21, são de maiores magnitude quando comparadas às características de reprodução. Portanto, a inclusão dessa característica na expressão da PAC torna-se essencial e deve ser considerada no critério de seleção de fêmeas. A maior produtividade nas fêmeas poderá ser atingida desde que a vaca produza progênies mais pesadas e com periodicidade, ou seja, maior porcentagem de bezerros nascidos e desmamados ao longo de sua vida útil, indicando a importância da taxa de prenhez e de desmama. E a expressão da PAC contempla todos esses aspectos ao considerar o peso do bezerro ao desmame e o número de progênies produzidas durante a permanência da fêmea no rebanho.

\section{CONCLUSÕES}

Os resultados encontrados no presente estudo sobre a PAC indicam a existência de variabilidade genética e a relevância de incluí-la nos programas de melhoramento, considerando que incorpora muitas características importantes na seleção de fêmeas.

$\mathrm{O}$ valor da estimativa de herdabilidade da PAC sugere a importância do seu uso na seleção de vacas, o que conduziria à obtenção de fêmeas mais produtivas, desmamando bezerros com mais peso (kg) por ano, porém o progresso genético seria lento.

\section{AGRADECIMENTOS}

Os autores agradecem aos criadores do Programa de Melhoramento Animal, à equipe técnica do PMGRN pela colaboração no desenvolvimento desta pesquisa e à FAPESP, $\mathrm{CNPq} / \mathrm{RHAE}$ pelo apoio financeiro.

\section{REFERÊNCIAS BIBLIOGRÁFICAS}

BITTENCOURT, T.C.C., LÔBO, R.B., REYS, A. de los, $\boldsymbol{e} \boldsymbol{t} \boldsymbol{a l}$. Uso da fertilidade real como medida de produtividade em rebanhos de bovinos de corte. In: SIMPÓSIO: O NELORE DO SÉCULO XXI, 4, 1997.Uberaba, MG. Anais... Uberaba : ABCZ, 1997. 244p. p.242.

BOLDMAN, K.G., KRIESE, L.A., VAN VLECK, L.D., $\boldsymbol{e}$ t al. A manual for use for MTDFREML. A set of programs to obtain estimates of variance and covariance [DRAFT] Lincoln : Department of Agriculture/ Agricultural Research Service, 1995. 120p.

DINKEL, C.A., BROWN, M.A. An evaluation of the ration of calf weight to cow weight as indication of cow efficiency. $\mathbf{J}$ Anim Sci, v.46, n.3, p.614-617, 1978.

EUCLIDES FILHO, K., RESTLE, J., OLSON, T.A., $\boldsymbol{e t}$ al. Medidas de eficiência na produção de terneiros a partir de vacas de tamanho e habilidade leiteira diferentes. In: REUNIAO ANUAL DA SOCIEDADE BRASILEIRA DE ZOOTECNIA, 21, 1984, Belo Horizonte. Anais... Belo Horizonte : SBZ, 1984. 503p. p.138.

FERRAZ FILHO, P.B., BIANCHINI SOBRINHO, E., SILVA, L.O.C. da et al. Estimativas de parâmetros genéticos e fenotípicos para pesos na raça Nelore mocha em três regiões Brasileiras. In: REUNIAO ANUAL DA SOCIEDADE BRASILEIRA DE ZOOTECNIA, 35, 1998, Botucatu. Anais... Botucatu : SBZ, 1998. 586p. p.517-519.

GARNERO, A del V. Comparação de critérios de seleção em gado de corte visando precocidade de crescimento. Ribeirão Preto, SP, 1999. 85p. Dissertação (Mestrado em Genética) - Faculdade de Medicina de Ribeirão Preto/Universidade de São Paulo, 1999.

KIRKPATRICK, B.W., DINKEL, C.A., RUTLEDGE, J.J. $\boldsymbol{e}$ t al Prediction equations of beef cow efficiency. J Anim Sci, v.60, n.4, p. 964-969, 1985.

LÔBO, R.B. Programa de melhoramento genético da raça Nelore. 3 ed. Ribeirão Preto : FINEP 1996. 104p.

MERCADANTE, M.E.Z. Estudo das relações genéticoquantitativas entre características de reprodução, crescimento e produção em fêmeas da raça Nelore. Ribeirão Preto, SP, 1995. 108p. Dissertação (Mestrado em Genética) - Faculdade de Medicina de Ribeirão Preto/Universidade de São Paulo, 1995.

NOTTER, D.R. Maximizing fertility in animal breeding programs. In: CONGRESSO BRASILEIRO DE REPRODUÇÃO ANIMAL, 11, 1995, Belo Horizonte. Anais... Belo Horizonte : Colégio Brasileiro de Reprodução Animal, 1995. 36p.

RAHNEFELD, G.W., WEISS, G.M., WARD, D. A comparison of methods to evaluate beef cow productivity. Can J Anim., v.73, n.4, p.971-975, 1993.

ROSA, A.N. Variabilidade fenotípica e genética do peso adulto e da produtividade acumulada de matrizes em rebanhos de seleção da raça Nelore no Brasil. Ribeirão Preto, SP, 1999. 114p. Tese (Doutorado em Genética) - Faculdade de Medicina de Ribeirão Preto/Universidade de São Paulo, 1999.

SAS Institute INC.SAS/STAT ${ }^{\mathrm{TM}}$ SAS user's guide for windowns environment. 6.11 ed. Cary : SAS Institute, 1995. 